\title{
Laser induced breakdown spectroscopy as diagnostics for fuel retention and removal and wall composition in fusion reactors with mixed- material components
}

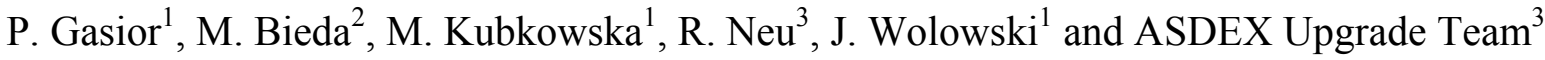 \\ ${ }^{1}$ Institute of Plasma Physics and Laser Microfusion / EURATOM Assoc.,ul. Hery 23, Warsaw, Poland \\ ${ }^{2}$ Faculty of Physics, Warsaw University of Technology, 75 Koszykowa, PL00662 Warsaw, Poland \\ ${ }^{3}$ Max-Planck-Institut für Plasmaphysik, EURATOM Assoc., D-85748 Garching, Germany
}

In order to apply Laser Induced Breakdown Spectroscopy (LIBS) for diagnostics of fuel retention and removal and wall composition in ITER it is necessary to validate the method in terms of repeatability and quality of its results for mixed, especially ITER relevant materials and its sensitivity to thin layers which contain small amounts of retained fuel.

The experiments, which are within the scope of this paper were carried out at the IPPLM Warsaw to assess the method for the laser treatment of ASDEX Upgrade (AUG) strike-point tiles and calibrated C:W:Al samples prepared by the Institute of Electronic Materials Technology (IEMT). The samples under investigation contained hydrogen isotopes in the ITER-relevant mixed material layer (as the Beryllium analogue, Al was used). Electrostatic ion energy analyzer was used as the auxiliary diagnostics.

The results proved that optical spectroscopy, although not straightforward in use, can give reliable results even for small amounts of fuel present in the mixed-material layer, although more effort should be put into investigating calibrated samples, to optimize the method itself and to yield a quantitative measure of fuel retention.

Keywords: Fuel removal, laser ablation, LIBS, PWI, first wall materials, ion diagnostics, pulsed lasers.

\section{Introduction}

Laser Induced Breakdown Spectroscopy (LIBS) [1] is one of the candidate methods for diagnostics of fuel retention and removal and wall composition in ITER. However, it is still questioned if its reliability and accuracy can meet the high requirements for diagnostics for the next-step fusion reactors. The main concerns lie in:

a) the repeatability and quality of the spectroscopic signal in stable and well-defined conditions (i.e. power density of delivered laser beam, plasma parameters),

b) applicability of the method to mixed material components, especially metals due to a high number of spectroscopic lines of them,

c) sensitivity of the method in the case of thin layers which contain small amounts of fuel.

All these issues, despite many experiments in different laboratories, have not been thoroughly investigated yet.

At the IPPLM the investigation of the LIBS has been carried out together with the optimization of the laser desorption [2,3] and ablation [4-10] methods for the removal of the layers co-deposited on the first wall components. After obtaining promising results for removal of thick co-deposites from TEXTOR limiter $[11,12]$, application of LIBS as real-time diagnostics for the process has been proposed and successfully tested [13].
Bearing in mind other promising experiments[14,15], the next step of the research was the extension of the method for its application in mixed material conditions. For this purpose, real-machine ASDEX Upgrade (AUG) strike-point tiles [16] and calibrated C:W:Al samples prepared by the Institute of Electronic Materials Technology, Warsaw (IEMT) by sputtering have been used as targets for laser irradiation. The choice of components in these samples was aimed to investigate spectroscopic response of various mixes of ITER relevant materials (with $\mathrm{Al}$ as the $\mathrm{Be}$ analogue). Both types of samples contained hydrogen isotopes on their surface. In the case of the AUG samples it was the result of plasma operation, and in case of the IEMT samples it was the result of hydrogen contamination in production process.

The first goal was to obtain repeatable and relatively high quality signals (with $\mathrm{S} / \mathrm{N}$ ratio better than 10 ) by proper choice of irradiation and signal collection parameters for single laser shots (a). Achieving this goal made it possible to focus on the behavior of optical spectra in evolving material mixes and in the presence of small amounts of hydrogen isotopes in very thin $(<0.5$ $\mu \mathrm{m})$ layers (b, c).

In the experiments presented in this contribution, LIBS was supported by auxiliary ion diagnostics (electrostatic ion energy analyzer [17] and ion collectors) for validation of the proper signal acquisition and the results in terms of identification of the chemical components detected in plasma. 


\section{Experimental set-up}

The detailed set-up of the experiment is presented in figure 1. Nd:YAG laser system was applied as the irradiation source. The laser delivered series of $3 \mathrm{~ns}, 0.6$ $\mathrm{J}$ laser pulses at $1.06 \mu \mathrm{m}$ at the repetition rate up to 10 $\mathrm{Hz}$. With the use of a lens the laser beam was focussed to the diameter of $\sim 3 \mathrm{~mm}$ at the target which resulted in the power density range of $10^{8} \mathrm{~W} / \mathrm{cm}^{2}$.

The applied spectrometer, Mechelle 5000 with IStar ICCD, made it possible to analyse the light of the wavelength range from 200 to $970 \mathrm{~nm}$ at wavelength accuracy of $\sim \pm 0.05 \mathrm{~nm}$ and the spectral resolution $(\lambda / \Delta \lambda)$ of 4000 . The emitted light was collected from the region of diameter of $\sim 3 \mathrm{~mm}$ located approximately $5 \mathrm{~mm}$ from the target.

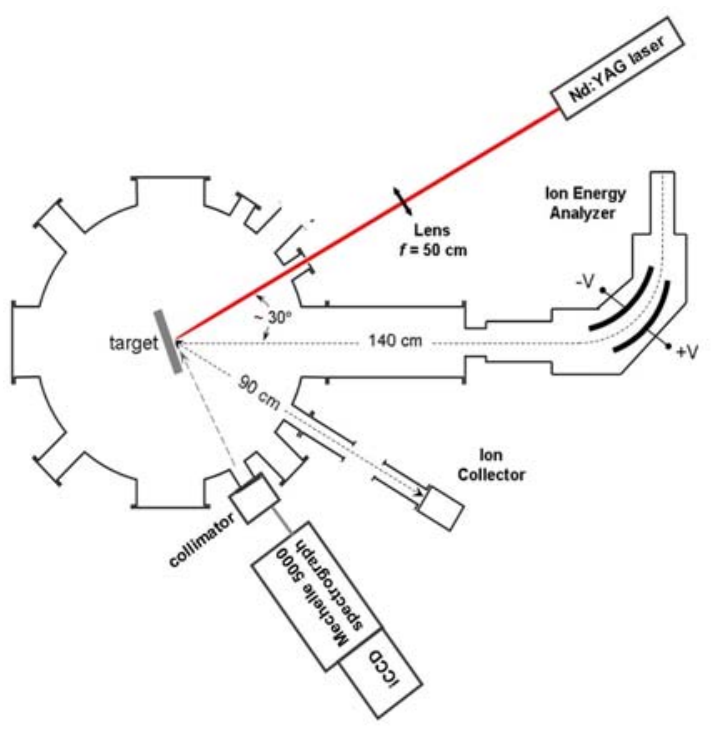

Fig.1 Experimental set-up of the LIBS device at IPPLM.

The target has been mounted in a vacuum chamber pumped down to the pressure of approximately $5 * 10^{-5}$ mbar.

Due to the thickness of the layer, which was comparable to the removal rate of the laser, the spectra were accumulated during only one or two laser shots in contrast to 5 laser shots performed in previous experiments on TEXTOR tiles [11]. Due to the short plasma life time and the need of investigation of the behavior of the material components during the temporal plasma evolution, the signal acquisition was triggered with delays of 20, 50,100,150, 200 and $300 \mathrm{~ns}$ after the laser shot and the gate was open for a part of a microsecond. The validity of the timing was confirmed by the signals from ion collectors.

The additional device was the electrostatic ion energy analyzer (IEA). Basing on the adjustment of the accelerating and deflecting potential of this device, it was possible not only to characterize the spectrum of the charged state of a given component extracted from the laser plasma, but also the chemical composition of laser treated samples. This feature was of the importance for the validation of the LIBS results. In the experiments the accelerating potential was set at $-4 \mathrm{kV}$ and the deflecting potential to \pm 80 and $\pm 100 \mathrm{~V}$ depending on the purpose of the experimental series.

Two general types of AUG samples were investigated - with 4 and $200 \mu \mathrm{m}$ tungsten coatings on graphite substrates produced by PVD (samples 04/1, a/1, i1) and VPS (sample 01b/1), respectively. The samples constituted parts mounted in the strike-point region of the AUG divertor. Most of the laser-treated surface was localized in regions where, codeposition/implantation of fuel ions was expected during AUG operation.

The samples provided by the IEMT contained thin layers of depth from 1 to $3 \mu \mathrm{m}$ which consisted of different mixtures of $\mathrm{C}: \mathrm{W}: \mathrm{Al}$. The layers were produced at the IEMT with the use of the sputtering technique.

\section{Result and discussion}

\subsection{AUG samples}

The presence of a deuterium line in LIBS spectra was recorded only for the first two laser shots in series for both types of samples. For the samples with thin coating, most tungsten was removed during the first 5 shots. The results in the spectral range, which is convenient for the $\mathrm{D} \alpha$ line observation are illustrated in figure 2 for a sample with the thin coating. The accumulated spectrum for the first two shots shows two distinct peaks of $\mathrm{D} / \mathrm{H}$ alpha line and tungsten. The amplitude of the deuterium line is significantly higher than amplitudes of carbon and tungsten in subsequent shots. It means that with the use of LIBS, the information on even small amount of fuel can be extracted from a spectrum which contains tungsten lines and was obtained at a low removal rate.

In the spectra of subsequent laser shots the tungsten lines become less significant due to the removal of the layer while carbon lines which are below the detection level for the first two shots become dominant.

For all samples, the magnitude of the deuterium line taken at the first laser shot was at the level of the highest tungsten line with $\pm 30 \%$ variation. In contrast to the high stability of the D $\alpha$ line, the lines of tungsten have not shown good repeatability but in each spectrum there were a few lines of high magnitude. This problem may be attributed to the complexity of the spectrum, which consists of many lines. On the other hand, the increase of delay of the recording, the contribution of lines corresponding to $\mathrm{W}^{0}$ increased in comparison to $\mathrm{W}^{+1}$ lines. This phenomenon is consistent with the lower propagation velocity of particles with lower charge to mass ratio.

The results of IEA were fully consistent with those obtained for LIBS. Results of the characterization of the deuterium content on the surface of the sample $04 / 1$ are shown in Figure $3 \mathrm{~A}$ )-C). The line attributed to deuterium is clearly seen in the signal collected for the first shot and its signature is still visible after the next shot. The deflecting potential suitable for observation of D line together with the $\mathrm{C}$ lines was $\pm 80 \mathrm{~V}$. 

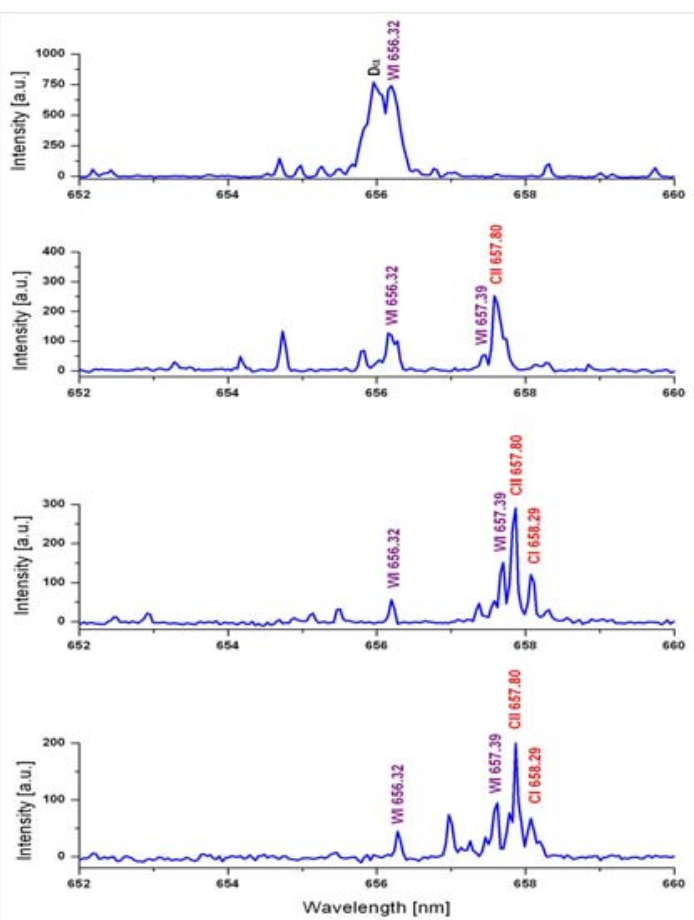

Fig.2 Evolution of optical spectra during the first 8 laser shots for AUG sample 04/1. Each subplot corresponds to the accumulated spectrum taken for two subsequent laser shots. The time order is from top to bottom.

For the subsequent shots the lines of carbon ions dominate the spectrum while the deuterium line is absent, which is consistent with the spectroscopic results.

Figure $3 \mathrm{D}$ shows the ion energy spectra taken for the higher deflecting voltage $( \pm 100 \mathrm{~V})$ after the 3rd laser shot in series. The spectra suggest that target composition consists of carbon and tungsten without any significant contaminants.
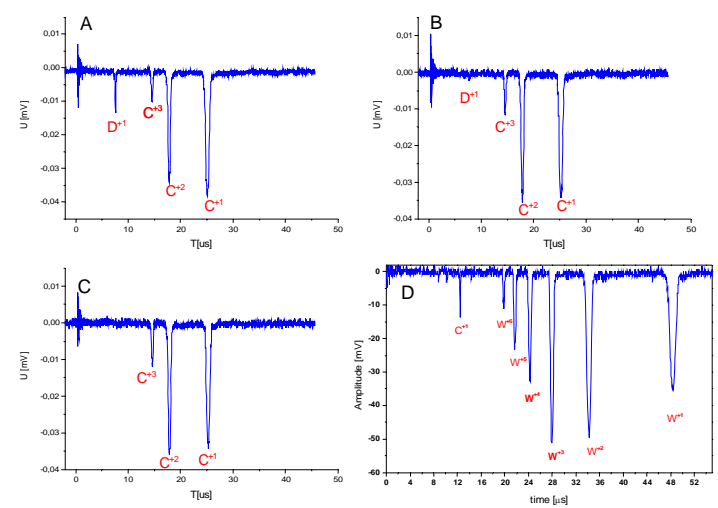

Fig. 3 Evolution of ion spectra during the first 3 laser shots for AUG sample 04/1 in the energy range suitable for $\mathrm{D}$ ion $(\mathrm{A}-\mathrm{C})$ and the overall spectrum for laser shot number 3 (D)

\subsection{Calibrated samples from IETM}

A number of calibrated samples with different mixes of ITER relevant materials have been investigated with the use of LIBS in the same set-up as for the AUG samples. One of the goals of these investigations was to measure spectroscopic signals corresponding to carbon, tungsten and aluminum for different mixtures of these components. For this purpose the concept of "synthetic contents coefficient" (SCC) was developed. The synthetic contents coefficient was the indicator of the contents of the spectroscopic signal corresponding to a given element in the overall spectroscopic signal of all plasma components. The coefficient was calculated with the use of the formula:

$$
S c C=\frac{\sum I_{I E}}{\sum I_{A E}}
$$

where $\sum I_{I E}$ is the sum of intensities of the representative lines of the element under consideration and $\sum I_{A E}$ is the sum of all the representative lines in spectra. Table 1 shows a set of mean values and standard deviations of Scc obtained for the components of three different samples based on statistic calculations applied for the results obtained in 6 series of irradiation under the same experimental conditions (time delay $150 \mathrm{~ns}$ and gate $500 \mathrm{~ns}$ ).

Table 1: Synthetic contents coefficient for the components of the different mixtures.

\begin{tabular}{ccrrr}
\hline \multirow{2}{*}{$\begin{array}{c}\text { Sample } \\
\text { Id. }\end{array}$} & $\begin{array}{c}\text { C:W:Al } \\
\text { ratio }\end{array}$ & \multicolumn{3}{c}{ Mean Value \pm Std. Deviation } \\
& \multicolumn{1}{c}{$S c c_{C}$} & \multicolumn{1}{c}{$S c c_{T}$} & \multicolumn{1}{c}{$S c c_{A l}$} \\
\hline \multirow{2}{*}{ VII } & \multirow{2}{*}{$1: 1: 1$} & 0,3689 & 0,3704 & 0,0953 \\
& & $\pm 0,0325$ & $\pm 0,0539$ & $\pm 0,0169$ \\
\hline \multirow{2}{*}{ VIII } & \multirow{2}{*}{$2: 1: 3$} & 0,3863 & 0,2249 & 0,3334 \\
& & $\pm 0,0487$ & 0,0193 & $\pm 0,0255$ \\
\hline \multirow{2}{*}{ IX } & \multirow{2}{*}{$3: 1: 2$} & 0,4363 & 0,1828 & 0,2794 \\
& & $\pm 0,0190$ & $\pm 0,0163$ & $\pm 0,0359$ \\
\hline
\end{tabular}

The values of the coefficient in the table are correlated with the ratio of the components in the mix, although the dependence does not seem to be straightforward. The standard deviation of the coefficients is in most cases lower than $10 \%$, which suggests that the measurement method and its parameters are reliable.

In figure 4 the results of table 1 are presented. The symbols show the values of the $\mathrm{S}_{\mathrm{cc}}$ obtained for single measurements on the 3 different targets (signed as VII, VIII and IX). The lowest scatter was obtained for sample IX. It may be attributed to the highest contents of Carbon in its layer, because Carbon has the lowest number of lines and the highest stability in comparison to the metallic components. The results also indicate that the coefficient for aluminum is most sensitive to changes in the mix and probably the matrix effect [1] depends more heavily on the ratio $\mathrm{Al}: \mathrm{W}$ than $\mathrm{Al}: \mathrm{C}$. On the other hand, the surprisingly low coefficient for the sample VII may be attributed to the lowest thickness of the layer.

Another experimental aim was to investigate the behavior of the $\mathrm{H} \alpha$ line during subsequent laser shots at samples with different material mixtures. As it had been expected, hydrogen line was quickly decreasing in intensity for all types of layers. The experimental results 
for samples VII, VIII and IX are presented in figure 5 where the obtained data points are shown together with the curves fitted to the exponential decay of the second order.

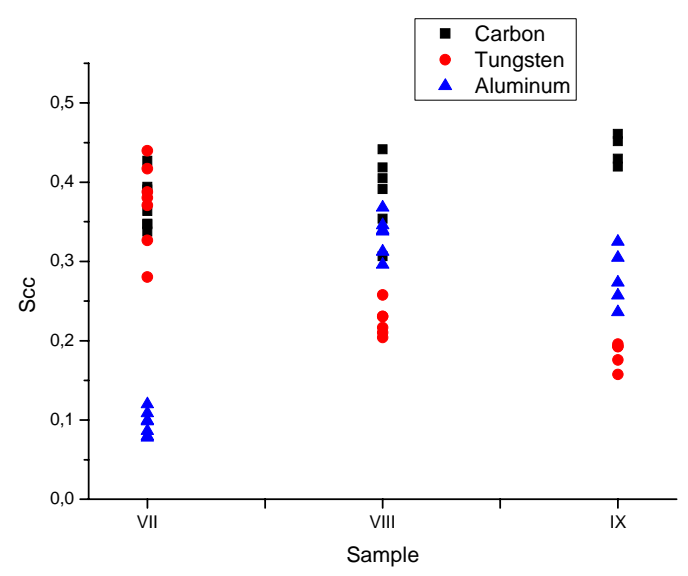

Fig.4 Synthetic contents coefficient for C, T and Al obtained for samples with various material mix (description in Table 1).

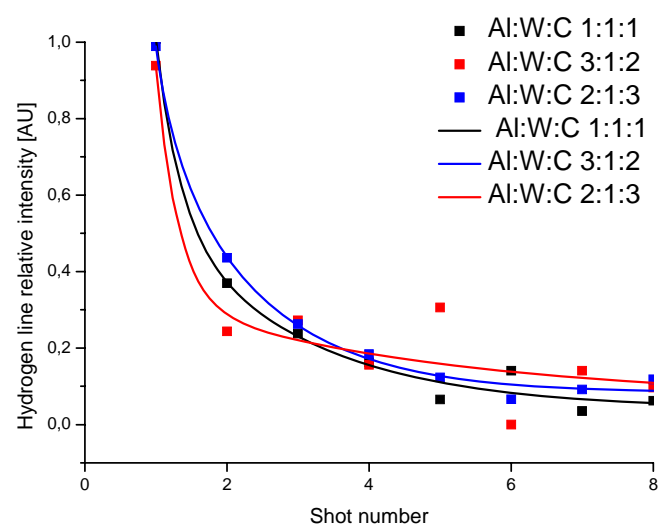

Fig.5 Experimental data for relative intensity of $\mathrm{H} \alpha$ line for layers with a different mix in dependence of the laser shot number and their fits.

The shapes of the fitting curves are very similar for all mixes, although, the fluctuation of datapoints, especially for the red line is visible. It suggests that LIBS diagnostics for measuring the contents of the hydrogen isotopes during the laser removal process may be relatively insensitive to the material mix of the layer in which retention takes place. The fluctuations observed for the red curve may be result from the local chemical nonuniformity of the layers which consists of materials with various hydrogen retention capabilities. To construe the results, one should also bear in mind, that total amount of the released hydrogen depends also on the removal rate of the layer.

\section{Summary and conclusion}

The results obtained for both, samples exposed in fusion devices and calibrated ones suggest that LIBS can be a very convenient method for detection and measurement of even small amounts of hydrogen isotopes released under laser treatment. The most important feature looks to be relatively independent from changes in the mix of the layer under investigation, at least for the layers with similar removal rate. In order to obtain good quantitative results still some experiments with precisely calibrated samples are needed, but the setup and the data interpretation methods do not need further enhancements.

Quantitative analysis of the chemical contents of the samples by LIBS is a more demanding task due to the complexity of the spectra of metallic components and cross-correlations between their synthetic contents coefficients, nevertheless, the results presented in this paper suggest that correlations between the amounts of the components and their content in the spectra are evident. Similarly as for quantitative measurements of the hydrogen isotope contents, further experiments on precisely calibrated samples are needed as well as analysis of the laser-treated surfaces by the means of materials research methods. These tasks are going to be a subject of the future experiments and studies at the IPPLM.

\section{Acknowledgment}

This work, supported by the European Communities under the contract of Association between EURATOM/IPPLM, was carried out within the framework of the European Fusion Development Agreement/EU PWI TF. The views and opinions expressed herein do not necessarily reflect those of the European Commission.

\section{References}

[1] D.A. Cremers \& L.J. Radziemski. Handbook of LaserInduced Breakdown Spectroscopy (London: John Wiley \& Sons, 2006),

[2] C.H. Skinner et al., J. Nucl. Mater. 301 (2002), p. 98-107,

[3] C.H. Skinner et al., Proceedings of the 17th IEEE/NPSS Symposium Fusion Engineering, San Diego, Ca. Oct 6-10, 1997, p.321,

[4] W.M. Shu, Y. Kawakubo, M.F. Nishi, Appl. Phys. A76 (2003) 421

[5] C.H. Skinner et al., J. Nucl. Mater. 313-316 (2003) 496.

[6] A. Semerok et al., J. Appl. Phys. 101 (2007) 084916.

[7] B. Emmoth et al., J. Nucl. Mater. 337-339 (2005) 639

[8] W.M. Shu et al., J. Nucl. Mater. 313-316 (2003) 584.

[9] C.H. Skinner et al., Phys. Scr. T103 (2003) 34.

[10] F. Le Guern et al., J. Nucl. Mater. 335 (2004) 3.

[11] P. Gąsior et al., Phys. Scr. T123 (2006) 99.

[12] P. Gąsior et al., Czech. J. Phys. 56 (2) (2006) B67.

[13] P. Gąsior et. al, J. Nuclear Mater. 390-391 (2009), pp.585588.

[14] C. Grisolia et al., J. Nucl. Mater. 363-365 (2007) 1138.

[15] F. Le Guern et al., Fus. Eng. Des. 81 (2006) 8.

[16] R. Neu et al., Phys. Scr. 2009014038

[17] Woryna, E., Parys, P., Wołowski, J., Mróz, W., Laser Part. Beams 14, 293 (1996). 